\title{
Triglyceride is an independent predictor of type 2 diabetes among middle-aged and older adults: a prospective study with 8-year follow-ups in two cohorts
}

Jing Zhao ${ }^{1+}$, Yuan Zhang ${ }^{1 \dagger}$, Fengjiang Wei ${ }^{1 \dagger}$, Jiani Song ${ }^{1}, Z_{\text {Zhi } \mathrm{Cao}^{2}, \text { Chen Chen }^{3} \text {, Kai Zhang }}{ }^{3}$, Shuzhi Feng ${ }^{3^{*}}$, Yaogang Wang ${ }^{2^{*}}$ and Wei-Dong $\mathrm{Li}^{1^{*}}$ (1)

\begin{abstract}
Background: Although there is abundant evidence indicating the connection between triglyceride and type 2 diabetes mellitus (T2DM), few reports or cohort studies confirm that high TG concentration may predict the incidence of T2DM independently. Thus, we studied the association between triglyceride (TG) and T2DM in a male-dominated, middle and older aged cohort, Tianjin General Hospital Cohort. And we further verified our results in the China Health and Retirement Longitudinal Study (CHARLS).

Methods: We conducted an 8-year retrospective cohort study (2009-2017) with 7241 participants who were free from T2DM at baseline. Three groups were constructed based on baseline TG levels (normal, borderline-high, and high). We used a Cox proportional hazards model to evaluate the relationship between TG and T2DM after adjusting for possible risk factors. A Kaplan-Meier survival analysis was performed to compare the incidence of T2DM among subjects in each TG group. We also tested the association between TG and T2DM in the CHARLS cohort.

Results: In Tianjin General Hospital Cohort, 7241 participants (male 75.8\%, female 24.2\%) were included, mean age was $61.49 \pm 13.85$ years at baseline. The cumulative incidence of T2DM in our cohort study was $8.6 \%(9.2 \%$ in men and $6.6 \%$ in women). Compared with the normal TG group, the hazard ratios in the borderline and high group were 1.30 (95\% Cl 1.04-1.62) and 1.54 (95\% Cl 1.24-1.90). The Kaplan-Meier survival analysis indicated that higher TG levels may predict higher onset of T2DM. These results were verified in the CHARLS cohort, the hazard ratio with T2DM (95\% Cl) for logTG was 3.94 (2.64-5.87).
\end{abstract}

Conclusions: Our findings suggest that the TG level may be an independent risk factor and predictor for T2DM. Keywords: Triglyceride, Type 2 diabetes mellitus, Dynamic cohort study, Survival analysis

*Correspondence: shuzhifeng@hotmail.com; wyg@tmu.edu.cn; liweidong98@tmu.edu.cn

${ }^{\dagger}$ Jing Zhao, Yuan Zhang and Fengjiang Wei contributed equally to this work

${ }^{1}$ Department of Genetics, College of Basic Medical Sciences, Tianjin Medical University, 22 Qixiangtai Road, Heping District, Tianjin 300070, People's Republic of China

${ }^{2}$ College of Public Health, Tianjin Medical University, 22 Qixiangtai Road, Heping District, Tianjin 300070, People's Republic of China

${ }^{3}$ Tianjin General Hospital, Tianjin Medical University, 154 Anshan Road, Tianjin 300052, People's Republic of China

\section{Background}

Diabetes mellitus (DM) affects $11.6 \%$ of the Chinese adult population [1]. It is predicted that there will be a $69 \%$ increase in prevalence of adult diabetes mellitus in developing countries between 2010 and 2030 [2]. DM patients have higher risks of cardiovascular disease, chronic kidney disease, cancer, and even death [3-5]. Thus, it is important to identify individuals who have higher risks for diabetes to facilitate early prevention and treatment. 
Triglycerides (TGs) are the most abundant lipid in human adipose tissue. Classification of TG level by NCEP Guide in 2001 is: desirable $<1.7 \mathrm{mmol} / \mathrm{L}$ (150 mg/dL), borderline-high 1.7-2.25 mmol/L (150$199 \mathrm{mg} / \mathrm{dL})$, high $2.26-5.6 \mathrm{mmol} / \mathrm{L}(200-499 \mathrm{mg} / \mathrm{dL})$ and very high $\geq 5.6 \mathrm{mmol} / \mathrm{L}(500 \mathrm{mg} / \mathrm{dL})$. In the United States, $31 \%$ adults have a TG level $\geq 1.7 \mathrm{mmol} / \mathrm{L}, 16.2 \%$ of adults exhibit a high $(\geq 2.26 \mathrm{mmol} / \mathrm{L}) \mathrm{TG}$ level, while $1.1 \%$ have very high $(\geq 5.6 \mathrm{mmol} / \mathrm{L}) \mathrm{TG}[6]$. Shown by a number of epidemiological studies, TG level was linearly correlated with the risk of T2DM [7-9]. Furthermore, a study indicated that among many lipid indexes which might be associated with T2DM, TG was the most significant [10]. Until now, some studies have been aimed at the relationship between TG level and T2DM in young men or women $[11,12]$. There are also some cross-sectional studies showing the link between TG and T2DM [13, 14]. But there are few reports or cohort studies which confirm that high TG concentration may predict the incidence of T2DM independently after adequately adjusting for potential confounders.

We collected a dynamic cohort, Tianjin General Hospital Cohort, consisting mainly of senior citizens who had regular physical examinations each year from 2009 to 2017 in Tianjin, China. By using Cox regression analysis, Kaplan-Meier survival analysis and other statistic method, We aim to reveal the association between TG and T2DM. We furthermore validated our result in another cohort, the China Health and Retirement Longitudinal Study (CHARLS).

\section{Materials and methods Study participants}

We collected 8956 subjects from Tianjin General Hospital Cohort who had annual comprehensive physical examinations from 2009 to 2017. Among these individuals, 1715 subjects were excluded due to prevalent T2DM or missing data in TG and fasting plasma glucose levels. A total of 7241 subjects were included in this study. All participants gave written informed consent prior the study, and the protocol was authorized by the Human Ethics Committee of Tianjin Medical University.

The China Health and Retirement Longitudinal Study (CHARLS) is a nationally representative longitudinal survey, investigating people of 45 years of age or older in China. We selected 17,500 people in CHARLS from 2011 to 2015 . Among these people, 5653 of them who lacked data of follow-up or suffered from DM were excluded. 2257 participants missed FPG or other covariates were excluded. Finally, a total of 9590 subjects were included to validate our result.

\section{Data collection}

Information of all the participants' physical examination are collected every year, from 2009 to 2017. Including glucose and lipid metabolism related index and some function related data, such as renal and liver function. All the measurements were performed by well-trained nurses or doctors. Body mass index (BMI) was calculated as weight in kilograms divided by height in meters squared. Blood pressure was measured after sitting for at least 5 min by standard mercury sphygmomanometer, and the average of two values reported. Blood samples were drawn from the antecubital vein after an overnight fast. Venous blood samples were used to measure serum uric acid (SUA) levels, lipid profiles (total cholesterol, TC; TG), fasting glucose, renal function (estimated glomerular filtration rate), liver function (alanine aminotransferase), hyperuricemia. TC and TG concentrations were measured by enzymatic calorimetric tests.

Information about CHARLS were downloaded from http://charls.pku.edu.cn/zh-CN after having permission.

\section{Definition}

T2DM was defined in subjects as fasting plasma glucose $\geq 7.0 \mathrm{mmol} / \mathrm{L}$ or subjects described T2DM history, and/ or if the subject is currently using antidiabetic medication. First occasion of documented T2DM per subject was recorded as the index event to calculate incidence rates. Hypertension was defined as the subject having a systolic blood pressure of greater than $140 \mathrm{~mm} / \mathrm{Hg}$ and/ or a diastolic blood pressure of greater than $90 \mathrm{~mm} / \mathrm{Hg}$ [15]. When an estimated glomerular filtration rate (eGFR) was observed to be $<60 \mathrm{~mL} / \mathrm{min} / 1.73 \mathrm{~m}^{2}$, the subject was defined to have chronic kidney disease. Hyperuricemia was defined when serum uric acid (SUA) $\geq 7.0 \mathrm{mg} / \mathrm{dL}$ $(420 \mu \mathrm{mL} / \mathrm{L})$ in males and SUA $\geq 6.0 \mathrm{mg} / \mathrm{dL}(360 \mu \mathrm{mL} /$ $\mathrm{dL})$ in females.

\section{Exposure data}

According to the TG concentration of participants in the baseline. Three groups were categorized in both Tianjin General Hospital Cohort and CHARLS by TG level: nor$\mathrm{mal}<1.7 \mathrm{mmol} / \mathrm{L}$, borderline high $1.7-2.25 \mathrm{mmol} / \mathrm{L}$, and high $\geq 2.26 \mathrm{mmol} / \mathrm{L}$.

\section{Statistical analyses}

The data for continuous variables used mean $\pm S D$ to describe while categorical variables used percentages (\%). First, we analyzed the basic characteristics in different groups at baseline in Tianjin General Hospital Cohort. In order to explore the relationship between TG and T2DM, a Cox proportional hazards model was established to evaluate hazard ratios (HR) and 95\% confidence intervals 
(CI) of the incidence of T2DM by TG levels. As TG level in population is not meet Gaussian distribution, we take logTG an index to Cox proportional hazards model to study the association between TG and T2DM. Based on TG concentration grouping, we used a Kaplan-Meier survival analysis to compare cumulative incidences of T2DM among the three groups, to see if high TG level accompany high cumulative incidence of T2DM. Further stratified analyses get $\mathrm{HR}$ and $\mathrm{CI}$ for the incidence of T2DM which were conducted to defined in various subgroups: age group $(<60, \geq 60$ years), sex (male, female), hypertension (yes, no), BMI $\left(<24,24-27.9, \geq 28 \mathrm{~kg} / \mathrm{m}^{2}\right)$, eGFR $\left(<60, \geq 60 \mathrm{~mL} / \mathrm{min} / 1.73 \mathrm{~m}^{2}\right)$, hyperuricemia (yes, no). Results of stratified analyses are tested by log-rank. All statistical analyses were performed using SPSS statistical software for Windows, version 17.0 (SPSS Inc., Chicago, IL, USA).

\section{Results}

The mean age of the 7241 participants was $61.49 \pm 13.85$ years, with 5492 men (75.8\%; $67.75 \pm 12.98$ years) and 1749 women $(24.2 \% ; 67.1 \pm 12.18$ years) at baseline. Table 1 showed the baseline characteristic of subjects in Tianjin General Hospital Cohort. Our results revealed that the individuals who had higher TG levels were likely to have higher FPG, BMI, SUA, ALT, and blood pressure.

The cumulative incidence of T2DM in Tianjin General Hospital Cohort was $8.6 \%$ (9.2\% in males; $6.6 \%$ in females; $X^{2}=11.254, P=0.001$ ) during the 8-year followup. Table 2 showed that $\log$ TG, hypertension, BMI, HUA, and sex were significantly associated with T2DM, while $\log$ TG was the most significant. The hazard ratio $(95 \%$ $C I)$ for $\log$ TG was 2.58 (1.87-3.57), while for hypertension, BMI, hyperuricemia, and female sex, these ratios were 1.88 (1.59-2.22), 1.11 (1.09-1.14), 1.34 (1.10-1.63), and $0.689(0.56-0.84)$. We tested the association between TG and T2DM with the China Health and Retirement Longitudinal Study (CHARLS). Table 2 showed that the hazard ratio with T2DM (95\% CI) for logTG was 3.94 (2.64-5.87). Hypertension, BMI, HUA, and sex were also associated with the risks of T2DM; the hazard ratios (95\% CI) were $1.37(1.11-1.69), 1.10(1.07-1.13), 0.64$ (0.42-0.96), and $0.81(0.65-1.00)$, respectively.

Our findings suggest that the correlation between TG and T2DM remained in all three models both in Tianjin General Hospital Cohort and CHARLS (Table 3). In Tianjin General Hospital Cohort, compared with the first group, in an unadjusted model, the hazard ratios for T2DM in the second and third groups were 1.42 (1.16$1.74)$ and 1.65 (1.36-2.00), respectively. After adjusting for age and sex, the hazard ratios in the second and third groups were 1.45 (1.18-1.78) and 1.75 (1.44-2.12). After further adjustment, the association remained significant. In model 3, the hazard ratios $(95 \% C I)$ of the second and third groups versus first group were 1.30 (1.04-1.6) and 1.54 (1.24-1.90), respectively. When the TG level was analyzed as a continuous variable, the association was still pronouncedly observed in model 1-3 between high TG level and T2DM. These results in CHARLS were consistent with our results, showing that TG level is associated with T2DM. In CHARLS, it showed that the correlation between TG and T2DM remained in all three

Table 1 Baseline characteristics of subjects in Tianjin General Hospital Cohort

\begin{tabular}{|c|c|c|c|c|}
\hline Characteristics & Normal (<1.7) & Borderline (1.7-2.25) & High $(\geq 2.26)$ & $P$-value* \\
\hline Male (\%) & 3639 (73.9\%) & $898(77.3 \%)$ & $955(82.5 \%)$ & $<0.001$ \\
\hline Age (years) & $62.05 \pm 14.27$ & $61.76 \pm 13.12$ & $58.83 \pm 12.36$ & $<0.001$ \\
\hline BMI $\left(\mathrm{kg} / \mathrm{m}^{2}\right)$ & $24.16 \pm 3.14$ & $25.36 \pm 2.29$ & $26.01 \pm 2.78$ & $<0.001$ \\
\hline Hypertension & $2015 / 4569$ (40.9) & $550 / 1065(47.3)$ & $539 / 1041(46.5)$ & $<0.001$ \\
\hline FPG (mmol/L) & $5.08 \pm 0.64$ & $5.16 \pm 0.66$ & $5.17 \pm 0.66$ & $<0.001$ \\
\hline eGFR-mL/min/1.732 & $88.72 \pm 19.60$ & $86.57 \pm 17.45$ & $87.26 \pm 23.18$ & $<0.001$ \\
\hline $\mathrm{TG}(\mathrm{mmol} / \mathrm{L})^{\dagger}$ & $1.09 \pm 0.32$ & $1.94 \pm 0.16$ & $3.39 \pm 1.67$ & $<0.001$ \\
\hline $\mathrm{TC}(\mathrm{mmol} / \mathrm{L})$ & $4.76 \pm 0.83$ & $5.05 \pm 0.86$ & $5.21 \pm 0.89$ & $<0.001$ \\
\hline $\begin{array}{l}\text { Urine dipstick result } \geq 1+\text { protein-no./ } \\
\text { total no (\%) }\end{array}$ & $140 / 4290(3.2)$ & $50 / 1058(3.8)$ & $41 / 1014(4.0)$ & 0.275 \\
\hline Hyperuricemia & $541 / 4919(11)$ & 234/1158 (20.1) & $347 / 1158(30)$ & $<0.001$ \\
\hline SUA ( $\mu \mathrm{mol} / \mathrm{L})$ & $319.74 \pm 76.37$ & $351.90 \pm 75.85$ & $375.08 \pm 79.58$ & $<0.001$ \\
\hline $\operatorname{ALT}(I U / L)$ & $21.75 \pm 17.69$ & $25.92 \pm 15.25$ & $28.59 \pm 12.15$ & $<0.001$ \\
\hline
\end{tabular}

Date are expressed as mean $\pm \mathrm{SD}, n(\%)$

$B M /$ body mass index, Hypertension, FPG fasting plasma glucose, $T G$ plasma triglyceride level, $T C$ total cholesterol, eGFR estimated glomerular filtration rate, hyperuricemia, SUA serum uric acid, $A L T$ alanine aminotransferase

${ }^{*} P<0.05$ was considered statistically significant

† Grouped based on TG level: normal < 150 mg/dL; borderline high 150-199 mg/dL; high 200-499 mg/dL 
Table 2 Hazard ratios of TG levels for T2DM in the Tianjin General Hospital Cohort and in the CHARLS

\begin{tabular}{|c|c|c|c|c|}
\hline & \multirow{2}{*}{$\begin{array}{l}\text { Tianjin General Hospital Cohort } \\
\operatorname{HR}(95 \% C l)^{\dagger}\end{array}$} & \multirow{2}{*}{$\begin{array}{l}\text { CHARLS } \\
\operatorname{HR}(95 \% C l)^{\dagger}\end{array}$} & \multicolumn{2}{|l|}{$P$-value* } \\
\hline & & & Tianjin General Hospital Cohort & CHARLS \\
\hline $\log T G$ & $2.58(1.87-3.57)$ & $3.94(2.64-5.87)$ & $<0.001$ & 0.001 \\
\hline Female & $0.69(0.56-0.84)$ & $0.81(0.65-1.00)$ & $<0.001$ & 0.046 \\
\hline Age (years) & $1.026(1.02-1.03)$ & $1.02(1.00-1.03)$ & $<0.001$ & 0.005 \\
\hline $\mathrm{BMI}\left(\mathrm{kg} / \mathrm{m}^{2}\right)$ & $1.11(1.09-1.14)$ & $1.10(1.07-1.13)$ & $<0.001$ & $<0.001$ \\
\hline Hypertension & $1.88(1.59-2.22)$ & $1.37(1.11-1.69)$ & $<0.001$ & 0.004 \\
\hline Hyperuricemia & $1.34(1.10-1.63)$ & $0.64(0.42-0.96)$ & $<0.001$ & 0.033 \\
\hline
\end{tabular}

TG plasma triglyceride level, $B M I$ body mass index

${ }^{*} P<0.05$ was considered statistically significant

${ }^{\dagger}$ Each time the value of hazard ratios changes by one unit, the risk doubles

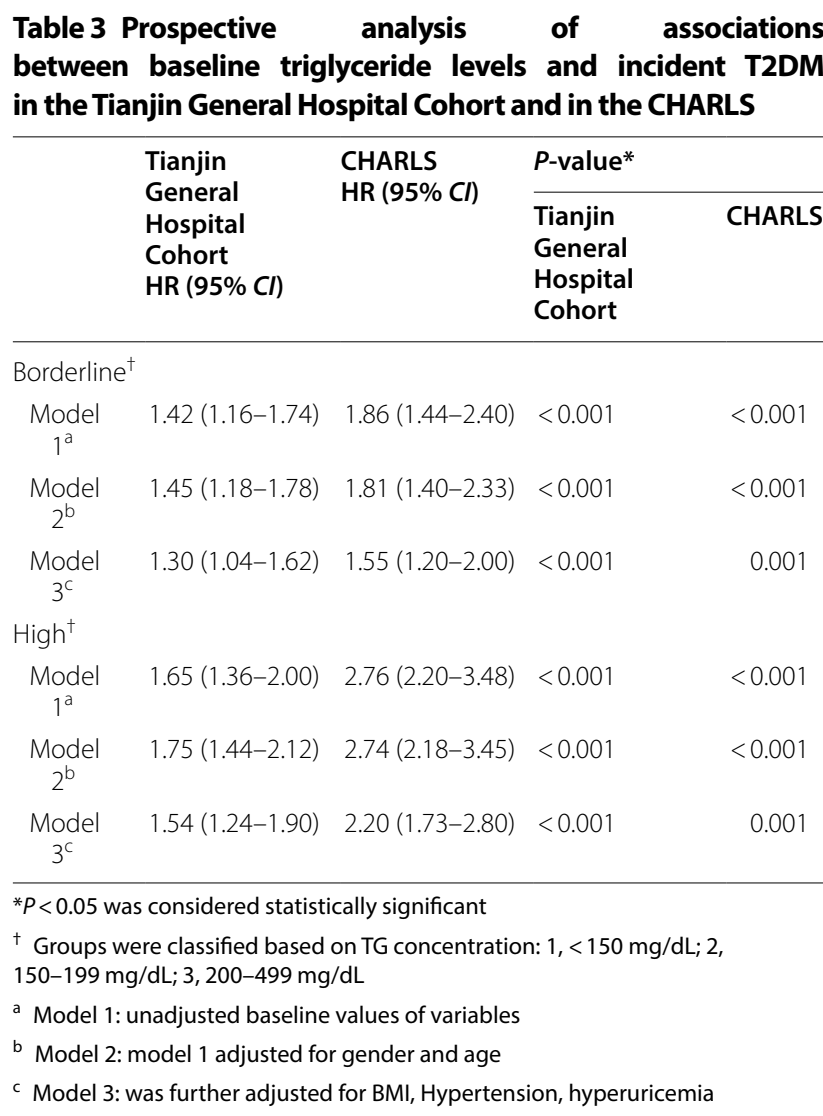

models. In an unadjusted model, compared with the first group, the hazard ratios in the second and third groups were $1.86(1.44-2.40)$ and $2.76(2.20-3.48)$. The hazard ratios were $1.81(1.40-2.33)$ and $2.74(2.18-3.45)$ after adjusting for age and sex in model 2 . In model 3 , the hazard ratios $(95 \% C I)$ of the second and third groups versus first group were $1.55(1.20-2.00)$ and 2.20 (1.73-2.80), respectively.

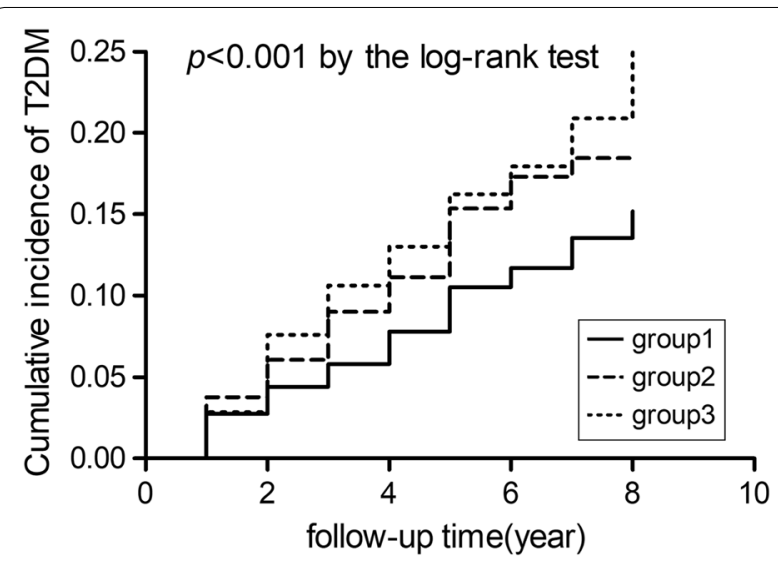

Fig. 1 Kaplan-Meier survival analysis K-M curves for T2DM among three TG levels. ${ }^{\dagger}$ Groups of TG were defined as follows: $1,<150 \mathrm{mg} / \mathrm{dL}$ (normal); 2, $150 \mathrm{mg} / \mathrm{dL}-199 \mathrm{mg} / \mathrm{dL}$ (borderline high); 3, 200-499 mg/ $\mathrm{dL}$ (high); ${ }^{*}$-value $<0.01$ for log-rank test

After testing the Kaplan-Meier survival analysis (Fig. 1) by log-rank test $(P<0.01)$, it showed that high TG levels were closely related to high incidence of T2DM in Tianjin General Hospital Cohort. The highest TG level showed the highest disease risk for T2DM among the three groups.

Moreover, subgroup analyses (Fig. 2) indicated that the association between TG level and T2DM was more evident in people who were younger than 60 years, of normal weight $\left(B M I<24.0 \mathrm{~kg} / \mathrm{m}^{2}\right)$, with abnormal eGFR, with hyperuricemia, and with no hypertension.

\section{Discussion}

Our results revealed that there was a significant linear association between TG levels and the incidence of T2DM. A higher TG level predicted higher hazard ratios of the presence of T2DM. This association was independent of age, sex, BMI, hypertension, and HUA, and it still remained significant after adjusting for other possible 


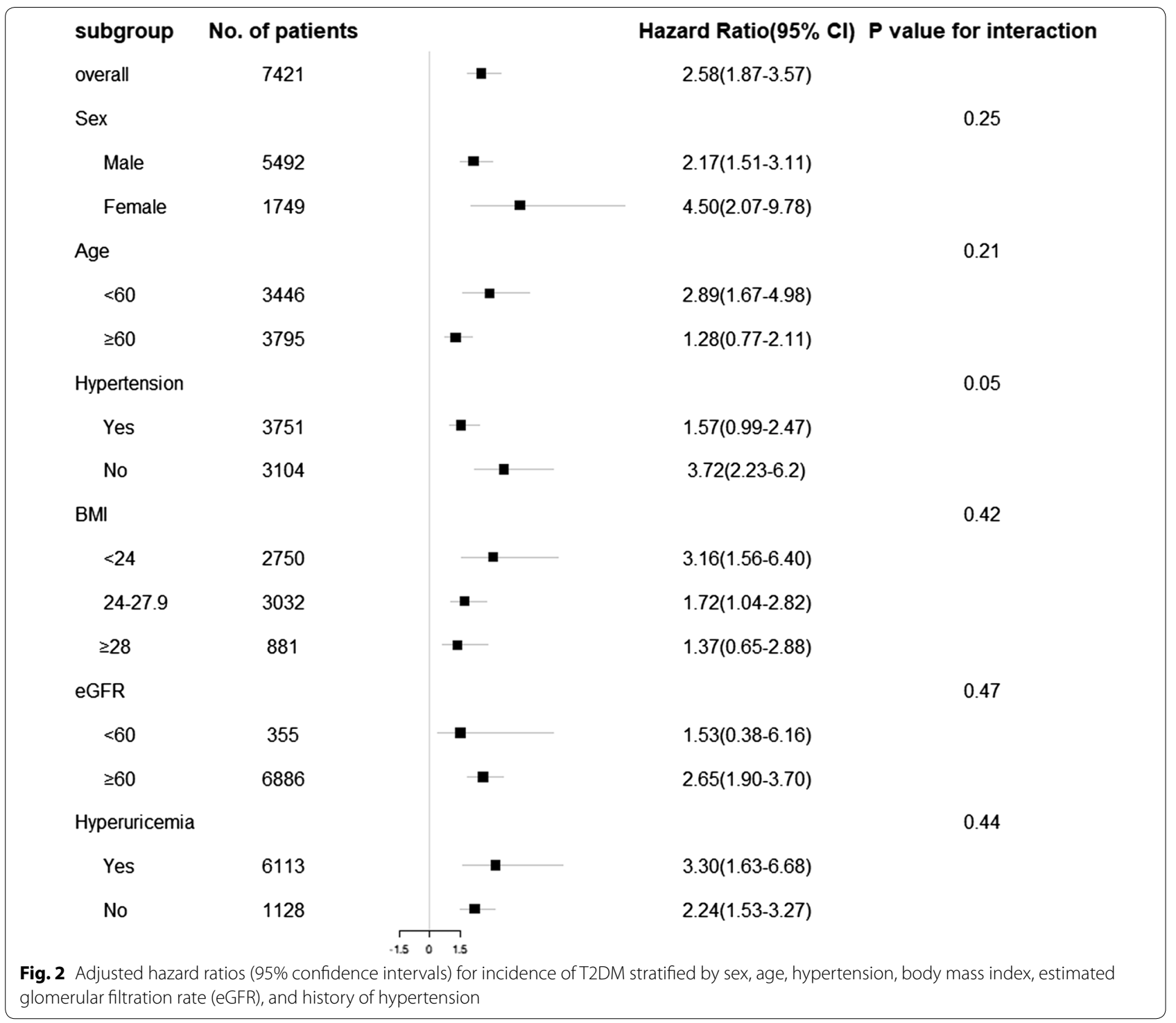

risk factors. The results showed that TG level may be a risk factor for T2DM.

A number of researches aimed to test whether the TG/HDL ratio could be a predictor of T2DM [10, 16, 17]. Different from previous studies, our study revealed that TG was an independent risk factor for diabetes and it might become an indicator of diabetes prediction. Our result was consistent with some earlier studies which revealed that TG level might be an influential factor for T2DM in children [18], young men [12], and women [11]. In our cohort study, we tested this relationship in an older population. Later, when this correlation (TG and T2DM) was tested in CHARLS, we were able to reach the same result. It indicated that high TG level may result in a high incidence of T2DM in the population of 45 years of age or older in China.
The cumulative incidence of T2DM in our cohort was $8.6 \%$ (9.2\% in men and 6.6\% in women), which was similar to the overall incidence in China. The overall pooled prevalence of diabetes was $8.3 \%$ (8.6\% in men and 8.0 in women) from 2010 to 2014 [19]. But a study based on 46,239 nationally representative samples showed that the age-standardized prevalence of total diabetes was 9.7\% [20]. One possible reason to explain the low prevalence of type 2 diabetes in our cohort could be attributed to the routine physical examination our subjects had each year, and may simply be relatively healthy part of the population. Another factor could be due to the likely higher social-economic status and higher income level these subjects have over the nationally representative group. A large population-based cross-sectional study in Tianjin found that people with low monthly income were 
associated with increased odds of T2DM [21]. It could support our results to a certain extent because the cohort we used were all from Tianjin as well.

The relationship between TG and T2DM risk was revealed in our study. Among numerous related factors, $\log$ TG has the biggest hazard ratios in diabetes mellitus. This was shown both in our original study and in CHARLS. Additionally, after controlling for confounding factors, borderline high and high TG levels were both correlated with an increased risk of T2DM. As an independent risk factor, high TG levels could be used as a guide for diabetes in clinical, which could make a more comprehensive prediction and assessment of health. People who have high TG levels are nearly $54 \%$ more likely to develop T2DM. The Kaplan-Meier survival analysis indicated that high TG levels may predict higher incidences of T2DM. In a previous cross-sectional study, the odds ratio was for T2DM was 1.87 (95\% CI 1.29-2.70) [22]; while the odds ratio in prediabetes and T2DM was 1.96 (95\% CI 1.422.69) and 3.91 (95\% CI 2.78-5.51) in a rural Bangladeshi study [23]. Both of these studies showed TG was a risk factor for T2DM although there are significant differences in numerical value. A cohort study [24] with 8867 subjects followed up for 5.5 years had a very similar result compared to ours. After adjusting for confounding factors, the hazard ratios for T2DM in the second, third and fourth quartiles were 1.07 (0.94-1.23), 1.17 (1.03-1.34), and 1.48 (1.30-1.69) when comparing with the first quartile. It showed the correlation between TG and T2DM.

Numerous factors may lead to T2DM including both congenital elements and acquired factors, but the specific mechanism remains unclear. Adipose tissue can influence glucose and lipid metabolism as an endocrine organ while TGs are the most abundant lipid in adipose tissue [25]. The potential mechanism linking TG to T2DM might refer the free fatty (FFA) metabolic pathways. As a large amount of FFA, resistin, TNF- $\alpha$, interleukin 6 and other compounds released by oversized adipose tissue can produce insulin resistance [26]. raising level of FFA produced by TGs can increase insulin resistance [27]. It has been already verified that the final outcome of insulin resistance is T2DM [28]. Furthermore, plasma FFA can enter cells easily and re-esterified for storage as TG, so raising TG levels may result in accumulation of TG $[26,29]$. In both Tianjin General Hospital Cohort and CHARLS indicated higher TG level accompany high risk of T2DM. Considering lifestyle and diet can influence TG levels, the physical mechanism between TG and T2DM need a further exploration under a large sample size, long follow-up and clinical trials.

Both strengths and limitations existed in our study. Strengths included a large number of samples, high event rates and a long-term follow-up (8 years). Many detailed clinical and biochemical assessments were included which allowed us to adjust for multiple potential confounders. After consulting numerous references, when known risk factors were controlled, our research is the first to report that TG level is a true independent risk factor associated with T2DM. Moreover, the same correlation between TG and T2DM in CHARLS supported this conclusion. It could provide a new approach to diagnose T2DM in clinical. As for limitations, one of the limitations was that the data of lifestyle choices such as drinking habits and smoking was not included in our study, which may affect the result [30,31]. Another limitation was that our subjects were "relatively healthy". These are people who had routine physical examinations and were not medical-seeking patients. While Tianjin General Hospital is a top tertiary care center, the subjects we included tended to be college teachers and/or staff of government-funded agencies. Our test population had relatively high socioeconomic status as a whole. Thus, our result might not represent the general population. As a strength, meanwhile, the long-term follow-up may be a limitation. Medicine testes were updated and new physical indicators were joined during the past 8 years, it made our cohort difficult to keep some indicators unbroken. We only have recent data for HbA1c (2014-2018) (Additional file 1: Table S2), the HbA1c level showed as an important indicator of T2DM $(\mathrm{HR}=3.49,3.21-3.80$, $P<0.001$ ) (Additional file 1: Table S3).

To conclusion, Both Tianjin General Hospital Cohort and CHARLS indicated that the increased TG level was significantly associated with incidence of T2DM. Higher TG level was associated with higher risk of T2DM independently. Survival analysis in Tianjin General Hospital Cohort showed that elevated TG level may predict higher cumulative incidence of T2DM. In order to decipher the relationship between TG and T2DM, future clinicals and basic research are needed.

\section{Supplementary information}

Supplementary information accompanies this paper at https://doi. org/10.1186/s12967-019-02156-3.

Additional file 1: Figure S1. Flow chart of subject selection for the present study. Figure S2. TG levels and the time to onset for T2DM. Table S1. Baseline characteristics of subjects in CHARLS. Table S2. Baseline characteristics of subjects in Tianjin General Hospital Cohort from 2014-2018. Table S3. Hazard ratios of TG levels for T2DM in the Tianjin General Hospital Cohort.

Acknowledgements

We thank all subjects who donated blood samples for genetic research purposes.

Authors' contributions

JZ designed the study, researched data and wrote the manuscript. YZ designed the study, researched data and contributed to discussion. W-DL designed the study, researched data and edited the manuscript. YW designed 
the study and contributed to discussion. FW researched data. JS researched data. ZC researched data. SF collected clinical data and contributed to discussion. CC, KZ collected clinical data and samples. All authors read and approved the final manuscript.

\section{Funding}

This work was supported by Grant 91746205 from the National Natural Science Foundation of China to Y.W.; and the National Key R\&D Program of China (2017YFC1001900).

\section{Availability of data and materials}

All the data and materials used in our article are available from the corresponding author on reasonable request.

\section{Ethics approval and consent to participate}

All participants gave written informed consent prior the study, and the protocol was authorized by the Human Ethics Committee of Tianjin Medical University.

\section{Consent for publication}

Not applicable.

\section{Competing interests}

The authors declare no competing interests.

Received: 21 August 2019 Accepted: 25 November 2019

Published online: 03 December 2019

\section{References}

1. Xu Y, Wang L, He J, Bi Y, Li M, Wang T, Wang L, Jiang Y, Dai M, Lu J, Xu M, Li Y, Hu N, Li J, Mi S, Chen CS, Li G, Mu Y, Zhao J, Kong L, Chen J, Lai S, Wang W, Zhao W, Ning G, China Noncommunicable Disease Surveillance G. Prevalence and control of diabetes in Chinese adults. JAMA. 2013;310(9):948-59.

2. Shaw JE, Sicree RA, Zimmet PZ. Global estimates of the prevalence of diabetes for 2010 and 2030. Diabetes Res Clin Pract. 2010:87(1):4-14

3. Gargiulo R, Suhail F, Lerma EV. Cardiovascular disease and chronic kidney disease. Dis Mon. 2015;61(9):403-13.

4. Leon BM, MaddoxTM. Diabetes and cardiovascular disease: epidemiology, biological mechanisms, treatment recommendations and future research. World J Diabetes. 2015;6(13):1246-58

5. Tirosh A, Rudich A, Shochat T, Tekes-Manova D, Israeli E, Henkin Y, Kochba I, Shai I. Changes in triglyceride levels and risk for coronary heart disease in young men. Ann Intern Med. 2007;147:377-85.

6. Miller M, Stone NJ, Ballantyne C, Bittner V, Criqui MH, Ginsberg HN, Goldberg AC, Howard WJ, Jacobson MS, Kris-Etherton PM, Lennie TA, Levi M, Mazzone T, Pennathur S, American Heart Association Clinical Lipidology T, Prevention Committee of the Council on Nutrition PA, Metabolism, Council on Arteriosclerosis T, Vascular B, Council on Cardiovascular N, Council on the Kidney in Cardiovascular D. Triglycerides and cardiovascular disease: a scientific statement from the American Heart Association. Circulation. 2011;123(20):2292-333.

7. Mc Donald Posso AJ, Bradshaw Meza RA, Mendoza Morales EA, Jaen Y, Cumbrera Ortega A, Mendoza Posada EJ. Diabetes in Panama: epidemiology, risk factors, and clinical management. Ann Glob Health. 2015;81(6):754-64.

8. Suvitaival T, Bondia-Pons I, Yetukuri L, Poho P, Nolan JJ, Hyotylainen T, Kuusisto J, Oresic M. Lipidome as a predictive tool in progression to type 2 diabetes in Finnish men. Metabolism. 2018;78:1-12.

9. Wang YL, Koh WP, Talaei M, Yuan JM, Pan A. Association between the ratio of triglyceride to high-density lipoprotein cholesterol and incident type 2 diabetes in Singapore Chinese men and women. J Diabetes. 2017;9(7):689-98.

10. Sung KC, Reaven G. Fasting plasma triglyceride concentration: a possible approach to identify increased risk of statin-induced type 2 diabetes. Diab Vasc Dis Res. 2015;12(5):373-6.

11. Norhammar A, Schenck-Gustafsson K. Type 2 diabetes and cardiovascular disease in women. Diabetologia. 2013;56(1):1-9.

12. Tirosh A, Shai I, Bitzur R, Kochba I, Tekes-Manova D, Israeli E, Shochat T, Rudich A. Changes in triglyceride levels over time and risk of type 2 diabetes in young men. Diabetes Care. 2008;31(10):2032-7.
13. Ozder A. Lipid profile abnormalities seen in T2DM patients in primary healthcare in Turkey: a cross-sectional study. Lipids Health Dis. 2014;13:183.

14. Thambiah SC, Samsudin IN, George E, Zahari Sham SY, Lee HM, Muhamad MA, Hussei Z, Mohd Noor N, Mohamad M. Relationship between dyslipidaemia and glycaemic status in patients with Type 2 diabetes mellitus. Malays J Pathol. 2016;38(2):123-30.

15. Gargiulo R, Suhail F, Lerma EV. Hypertension and chronic kidney disease. Dis Mon. 2015;61(9):387-95.

16. Cheng C, Liu Y, Sun X, Yin Z, Li H, Zhang M, Zhang D, Wang B, Ren Y, Zhao Y, Liu D, Zhou J, Liu X, Liu L, Chen X, Liu F, Zhou Q, Hu D. Dose-response association between the triglycerides: High-density lipoprotein cholesterol ratio and type 2 diabetes mellitus risk: The rural Chinese cohort study and meta-analysis. J Diabetes. 2019;11:183-92.

17. Zhou M, Li Z, Min R, Dong Y, Sun Q, Li Y. Log (TG)/HDL-C ratio as a predictor of decreased islet beta cell function in patients with type 2 diabetes: 6-year cohort study. J Diabetes. 2015;7(5):689-98.

18. Habiba NM, Fulda KG, Basha R, Shah D, Fernando S, Nguyen B, Xiong Y, Franks SF, Matches SJ, Magie RD, Bowman WP. Correlation of lipid profile and risk of developing type 2 diabetes mellitus in 10-14 year old children. Cell Physiol Biochem. 2016;39(5):1695-704.

19. Han C, Zhang M, Luo X, Wang C, Yin L, Pang C, Feng T, Ren Y, Wang B, Zhang L, Li L, Yang X, Zhang H, Zhao Y, Zhou J, Xie Z, Zhao J, Hu D. Secular trends in the prevalence of type 2 diabetes in adults in China from 1995 to 2014: a meta-analysis. J Diabetes. 2017;9(5):450-61.

20. Yang W, Lu J, Weng J, Jia W, Ji L, Xiao J, Shan Z, Li J, Tian H, Ji Q, Zhu D, Ge J, Lin L, Chen L, Guo X, Zhao Z, Li Q, Zhou Z, Shan G, He J. Prevalence of diabetes among men and women in China. N Engl J Med. 2010;362(12):1090-101.

21. Zhang H, Xu W, Dahl AK, Xu Z, Wang HX, Qi X. Relation of socio-economic status to impaired fasting glucose and Type 2 diabetes: findings based on a large population-based cross-sectional study in Tianjin, China. Diabet Med. 2013;30(5):e157-62.

22. Janghorbani M, Amini M. Utility of hypertriglyceridemic waist phenotype for predicting incident type 2 diabetes: the Isfahan Diabetes Prevention Study. J Diabetes Investig. 2016;7(6):860-6.

23. Bhowmik B, Siddiquee T, Mujumder A, Afsana F, Ahmed T, Mdala IA, do Moreira VNC, Khan AKA, Hussain A, Holmboe-Ottesen G, Omsland TK. Serum lipid profile and its association with diabetes and prediabetes in a rural Bangladeshi population. Int J Environ Res Public Health. 2018;15(9):E1944.

24. Fujihara K, Sugawara A, Heianza Y, Sairenchi T, Irie F, Iso H, Doi M, Shimano $\mathrm{H}$, Watanabe $\mathrm{H}$, Sone $\mathrm{H}$, Ota $\mathrm{H}$. Utility of the triglyceride level for predicting incident diabetes mellitus according to the fasting status and body mass index category: the Ibaraki Prefectural Health Study. J Atheroscler Thromb. 2014;21(11):1152-69.

25. Scherer PE. Adipose tissue: from lipid storage compartment to endocrine organ. Diabetes. 2006;55(6):1537-45.

26. Boden G. Obesity and free fatty acids. Endocrinol Metab Clin North Am. 2008;37(3):635-46, viii-ix.

27. Boden G, Chen X, Ruiz J, White JV, Rossetti L. Mechanisms of fatty acidinduced inhibition of glucose uptake. J Clin Invest. 1994;93(6):2438-46.

28. Fernandez-Real JM, Pickup JC. Innate immunity, insulin resistance and type 2 diabetes. Diabetologia. 2012;55(2):273-8.

29. Boden G, Lebed B, Schatz M, Homko C, Lemieux S. Effects of acute changes of plasma free fatty acids on intramyocellular fat content and insulin resistance in healthy subjects. Diabetes. 2001;50(7):1612-7.

30. Huang Y, Li J, Zhu X, Sun J, Ji L, Hu D, Pan C, Tan W, Jiang S, Tao X, Investigators C-BS. Relationship between healthy lifestyle behaviors and cardiovascular risk factors in Chinese patients with type 2 diabetes mellitus: a subanalysis of the CCMR-3B STUDY. Acta Diabetol. 2017:54(6):569-79.

31. Ma RCW, Lin X, Jia W. Causes of type 2 diabetes in China. Lancet Diabetes Endocrinol. 2014:2(12):980-91.

\section{Publisher's Note}

Springer Nature remains neutral with regard to jurisdictional claims in published maps and institutional affiliations. 